\title{
Comparison of mode suppression and large signal modulation between lattice matched and strained InGaAs/AIGaAs quantum well lasers
}

\author{
Yeeloy Lam, John P. Loehr, a) and Jasprit Singh \\ Center for High-Frequency Microelectronics and Solid State Electronics Laboratory, \\ Department of Electrical Engineering and Computer Scicnce, The University of Michigan, Ann Arbor, \\ Michigan 48109-2122
}

(Received 28 June 1991; accepted for publication 12 August 1991)

\begin{abstract}
Numerical techniques are developed to study the output spectra and to solve the coupled mode rate equations for $\mathrm{In}_{x} \mathrm{Ga}_{1-x} \mathrm{As} / \mathrm{Al}_{0.3} \mathrm{Ga}_{0.7}$ As quantum well lasers. The optical properties of the laser are calculated from a $4 \times 4 \mathrm{k} \cdot \mathrm{p}$ band structure which includes the effects of strain. Wo find that the side modes are severely suppressed in the strained laser. Large signal switching of the laser is also studied and the role of strain is identified in the device response. If the laser is switched from the off state to a state of given photon density in the lasing mode, then the strained system exhibits a faster time response. If, however, the laser is switched from the off state to a state of given total photon density, then the strained system has a slower time response.
\end{abstract}

In the last several years there has been a great deal of interest in pseudomorphic quantum well lasers. ${ }^{1-4} \mathrm{~A}$ number of forces are driving this interest. These range from lower threshold current, possibly reduced Auger rates, greater tunability in the wavelength, and possibly improved modulation speeds. ${ }^{5,6}$ In this letter we address two important issues in the $\operatorname{In}_{x} \mathrm{Ga}_{1-x} \mathrm{As} / \mathrm{Al}_{0.3} \mathrm{Ga}_{0.7} \mathrm{As}$ quantum well lasers: (i) the effect of strain on side-mode suppression in a Fabry-Perot laser and (ii) large signal modulation of the laser from operating points below threshold to above threshold. Rather than using phenomenological functions to describe the gain and differential gain function or the spontaneous emission rate we calculate these numerically using the Fermi Dirac distribution function. We also solve the multimode coupled rate equation including up to 250 modes.

Using a four-band k•p model to describe the confined valence band states in the strained quantum well, the subband to subband optical gain in the laser is calculated. ${ }^{4}$

We assume a typical Fabry-Perot resonator for the semiconductor laser with cavity length $L$ and track the photon output of each allowed Fabry-Perot cavity mode by using the multimode rate equations

$$
\begin{aligned}
& \frac{d S_{m}}{d t}=\left[\Gamma g\left(n, E_{m}\right)-\alpha_{c}\right]\left(\frac{c}{n_{g}}\right) S_{m}+\beta R \operatorname{sp}(n) \\
& \frac{d n}{d t}=\frac{J}{e}-R \operatorname{sp}(n)-\left(\frac{c}{n_{g}}\right) \sum_{m} \Gamma g\left(n, E_{m}\right) S_{m}
\end{aligned}
$$

where $S_{m}$ represents the photon population in the cavity for the $m$ th longitudinal mode oscillating at frequency $\omega_{m}$ and photon energy $E_{m}, \Gamma$ is the confinement factor, $g\left(n, E_{m}\right)$ is the material gain for the $m$ th mode photon population at carrier concentration $n, \alpha_{c}$ is the cavity loss, $n_{g}$ is the group index in the cavity, $\beta$ is the spontaneous emission factor, and $R_{\mathrm{sp}}(n)$ is the spontaneous emission rate at carrier concentration $n . J$ is the injected radiative

\footnotetext{
${ }^{\text {a) }}$ Present address: WL-ELRD, Wright Patterson AFB, Dayton, $\mathrm{OH}$ 45433-6503.
}

current density. We include all photon modes $E_{m}$ that exhibit positive gain at threshold.

The steady-state solution to the rate Eqs. (1) and (2) is obtained by setting all the time derivatives to zero. The carrier concentration that satisfies a given steady-state injected current is now obtained by iterative self-consistent solutions of the two coupled equations. To obtain transient solutions to the rate equations we solve Eqs. (1) and (2) numerically using a fourth-order Runge-Kutta algorithm. A number of simplifying assumptions are made in the calculations such as (1) the photon and electron (and hole) distributions are spatially uniform, (2) the refraction index is spatially uniform and the effect of its variations with time is neglected, (3) the optical confinement factor $\Gamma$ and the spontaneous emission factor $\beta$ are treated as constant, and (4) nonlinear photon effects (gain compression) are ignored.

Steady-state and transient numerical calculations are performed for (a) $\mathrm{GaAs} / \mathrm{Al}_{0.3} \mathrm{Ga}_{0.7} \mathrm{As}$ (lattice matched) and (b) $\mathrm{In}_{0.2} \mathrm{Ga}_{0.8} \mathrm{As} / \mathrm{Al}_{0.3} \mathrm{Ga}_{0.7}$ As (strained) $50 \AA$ width, $300 \mu \mathrm{m}$ length quantum well lasers. For a single $50 \AA$ quantum well laser we have used $\Gamma=1.25 \times 10^{-2}$. The optical confinement factor $\Gamma$ is also considered for spontaneously emitted photons but it is implicitly contained as one of the parameters contributing to the spontaneous emission factor $\beta$ in Eq. (1). ${ }^{7,8}$ Our calculation assumes a value of $\beta=10^{-5}$. We obtain a cavity loss $\alpha_{c}=1 / L \ln 1 / R+\alpha_{0}$ of $48 \mathrm{~cm}^{-1}$ for our structure by assuming an absorption loss $\alpha_{0}=10 \mathrm{~cm}^{-1}$ and mirror reflectivity $R=0.32$.

We calculated the steady-state spectral response for the lattice matched and strained lasers. We note that compressive strain of $1.4 \%$ reduces threshold current by roughly a factor of three. In addition, we find that side modes are greatly suppressed by the introduction of strain. Also, we find that when both lasers are essentially exhibiting single mode characteristics the strained quantum well (QW) laser produces about $30 \%$ more photon output than its lattice-matched counterpart. This difference becomes even greater at a lower current density, where the lattice- 

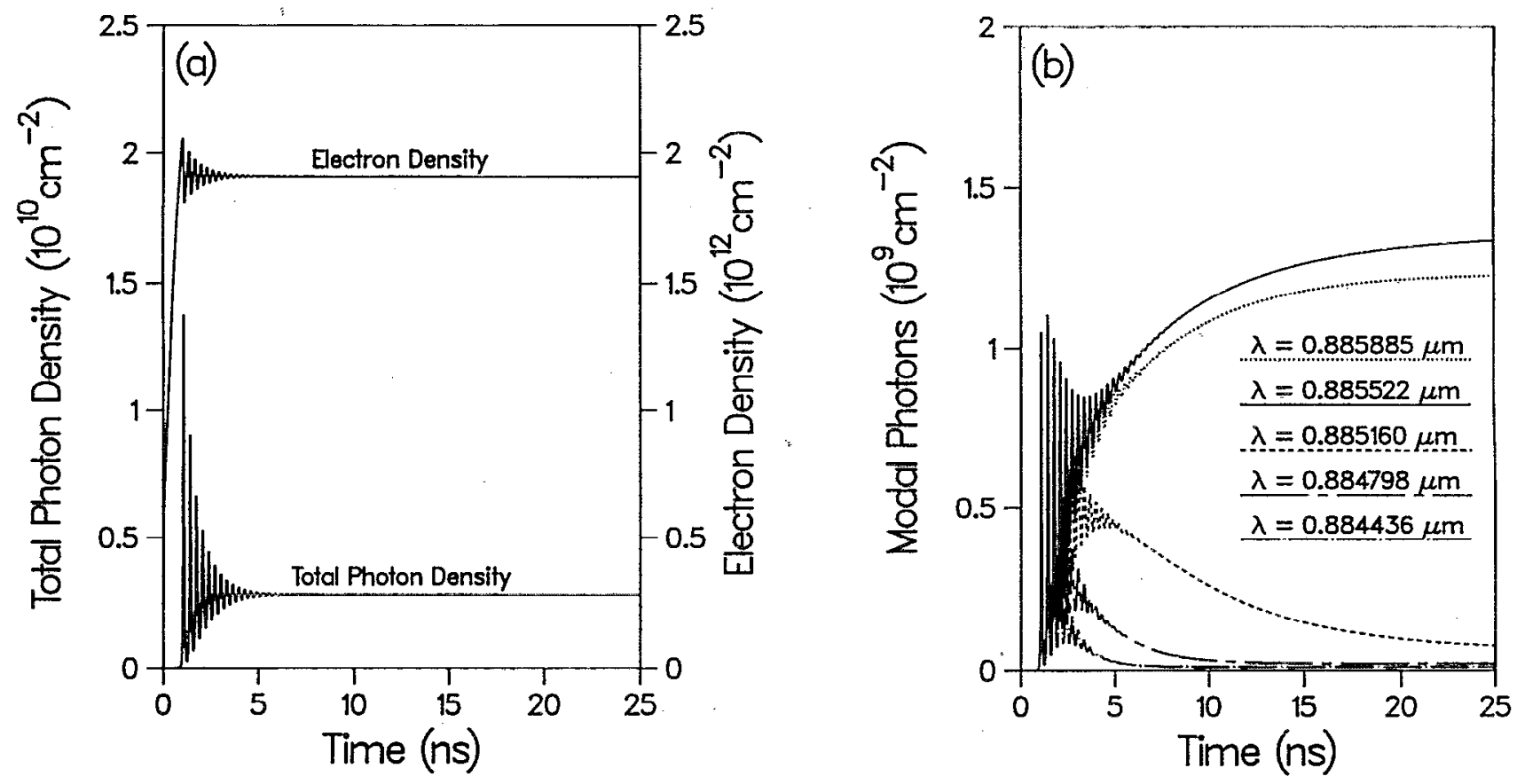

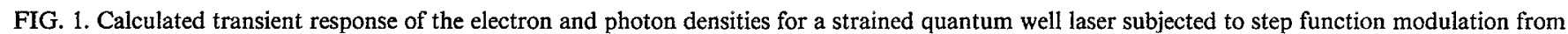
zero to twice threshold current density $\left(360 \mathrm{~A} / \mathrm{cm}^{2}\right)$. In (b) only 5 modes from the full spectrum are plotted.

matched laser is under multimode operation and the strained laser emits single mode.

In the transient calculation we subject the strained laser to an ideal step function signal modulation with the injected current changing from zero to twice its threshold current value $\left(360 \mathrm{~A} / \mathrm{cm}^{2}\right)$. The calculated transient response for the total photon density and the electron density for the strained QW laser are shown in Fig. 1(a). The response behavior as displayed in this figure appears rather similar to that calculated by Marcuse and Lee ${ }^{9}$ using a Lorentzian gain spectra. In Fig. 1(b), however, our numerical solution explicitly shows that the relaxation oscillations decay in a much shorter time than the time required to redistribute the optical power in the longtiudinal modes. We note from Fig. 2(a) that the envelope of the relaxation oscillations decays roughly exponentially with a time con-
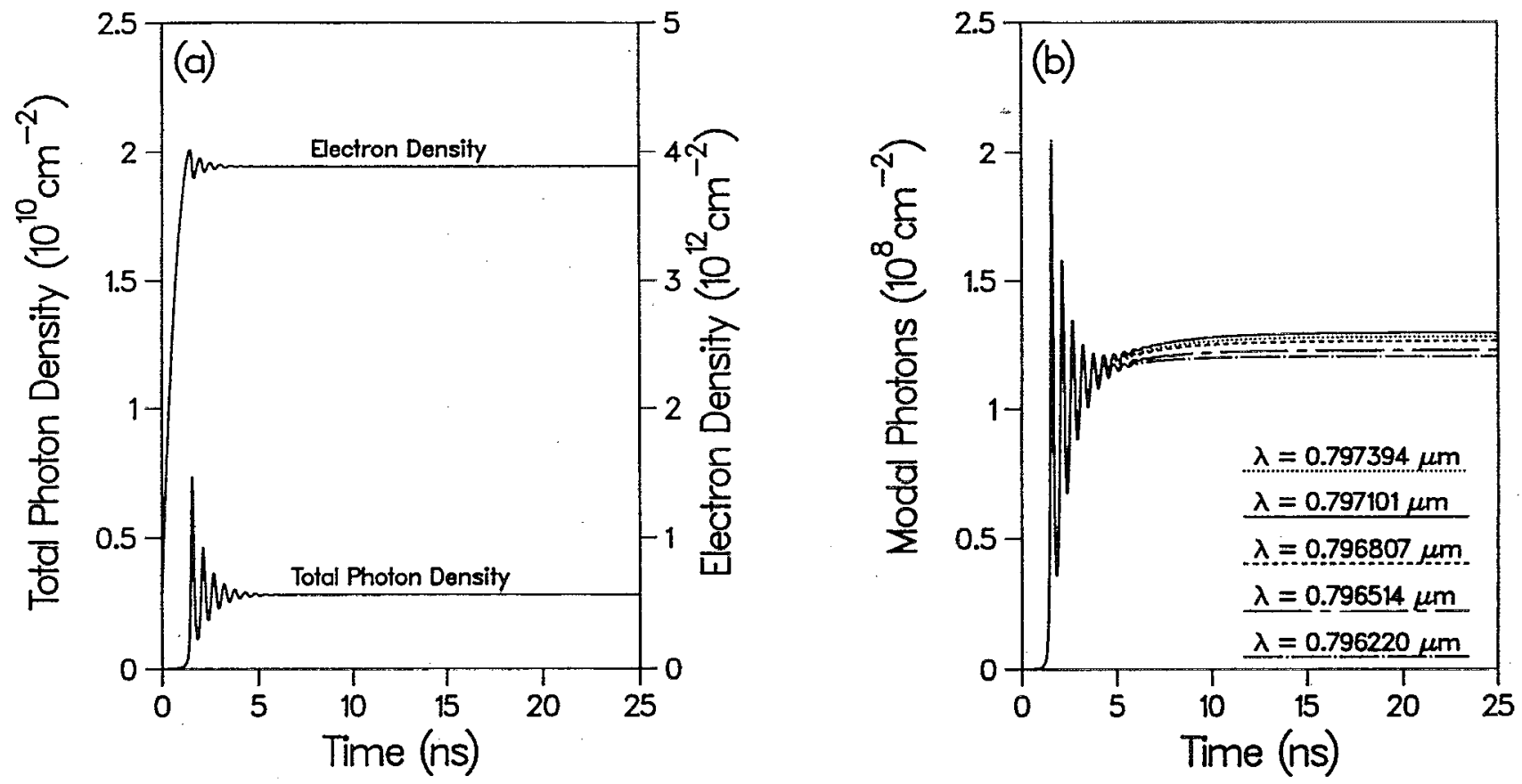

FIG. 2. Calculated transient response of the electron and photon densities for a lattice-matched quantum well laser subjected to step function modulation of current density from zero to $757 \mathrm{~A} / \mathrm{cm}^{2}$. In (b) only 5 modes from the full spectrum are plotted. 

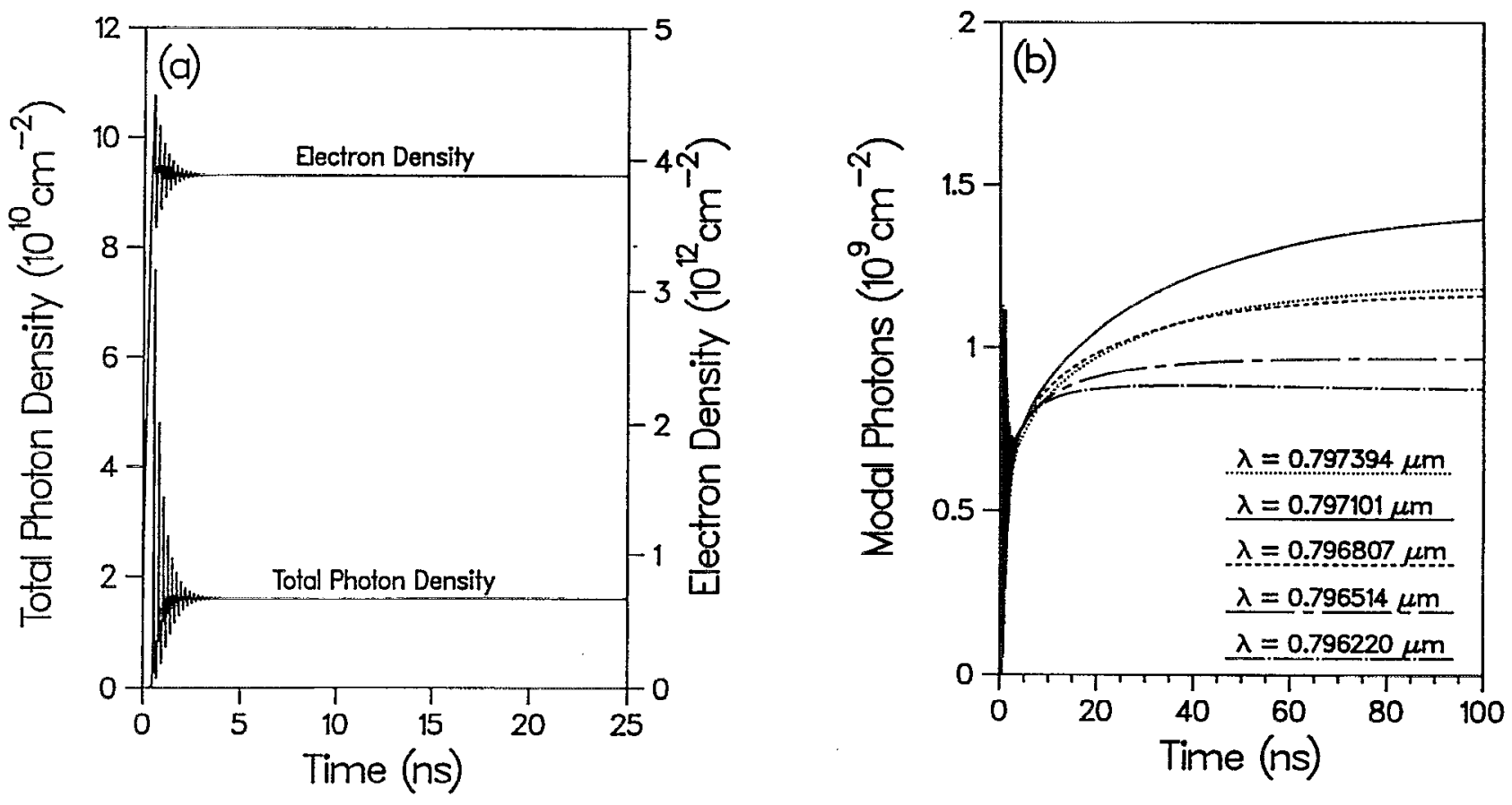

FIG. 3. Calculated transient response of the electron and photon densities for a lattice matched quantum well laser subjected to step function modulation of current density from zero to $1610 \mathrm{~A} / \mathrm{cm}^{2}$. In (b) only 5 modes from the full spectrum are plotted.

stant of about 3 ns, while Fig. 2(b) shows that the modal photon densities redistribute themselves in about $20 \mathrm{~ns}$, or about six times longer. Hence, we see that the strained laser is characterized by two dramatically different response times: a fast time ( $\approx 3 \mathrm{~ns}$ ) during which the total photon density equilibrates, and a much slower time $(\approx 20$ ns) during which the lasing mode photon density reaches its steady-state value.

To find out the role of strain, we contrast the results in Fig. 1 with two different calculations for the lattice matched laser. In the first case we switch the laser from zero current to a current value at which the steady-state total photon density is equal to that of the strained laser in Fig. $1\left(2.8 \times 10^{9} \mathrm{~cm}^{2}\right)$. This corresponds to a current of $757 \mathrm{~A} / \mathrm{cm}^{2}$ and we show the results in Fig. 2. In the second case we switch the laser to a current value where the steady-state photon density in the peak mode is the same as that in the strained laser case $\left(1.4 \times 10^{9} \mathrm{~cm}^{2}\right)$. This corresponds to a current of $1610 \mathrm{~A} / \mathrm{cm}^{2}$, and we show the results in Fig. 3.

We find from a comparison of Figs. 1 (b) and 2(b) that the lasing mode in the lattice-matched system reaches steady state faster than the strained laser, while the times taken to reach a given steady-state total photon density are similar. When we compare Figs. 1(b) and 3(b), however, we find that the time taken to reach a given steady-state lasing mode photon density is much longer for the lattice matched system. We see that the steady state in the peak mode is reached at approximately $25 \mathrm{~ns}$ in the strained case versus approximately $100 \mathrm{~ns}$ in the lattice matched case. It may be noted from the comparison of Figs. 1(a) and 3(a) that the total photon density reaches steady state faster in the lattice matched case. Of course, a lattice-matched sys- tem requires a much larger drive current to produce the same lasing mode photon density as the strained system $\left(1610 \mathrm{~A} / \mathrm{cm}^{2}\right.$ versus $\left.360 \mathrm{~A} / \mathrm{cm}^{2}\right)$.

From our results above, it is clear that for large signal modulation the main advantages of the strained laser are in systems where only peak mode photons are used in the transmission and detection. For cases where the communication involves the total photon density in all modes, the lattice matched system is intrinsically as fast as the strained system. Of course, in all cases the lower current required to drive the switching of strained lasers will reduce extrinsic time constant and heat dissipation problems.

This work was supported by a program from the Office of Naval Research No. N00014-90-J-1831.

${ }^{1}$ W. D. Laidig, P. J. Caldwell, Y. F. Lin, and C. K. Peng, Appl. Phys. Lett. 44, 653 (1984).

${ }^{2}$ P. J. A. Thijs and T. Van Dongen, Electron. Lett. 25, 1735 (1989).

${ }^{3}$ U. Koren, M. Oron, M. G. Young, B. I. Miller, J. L. De Miguel, G. Raybon, and M. Chien, Electron. Lett. 26, 465 (1990).

${ }^{4}$ J. P. Loehr and J. Singh, IEEE J. Quantum Electron. 27, 708 (1991).

${ }^{5}$ I. Suemune, L. A. Coldren, M. Yamanishi, and Y. Kan, Appl. Phys. Lett. 53, 1378 (1988).

${ }^{6}$ S. D. Offsey, W. J. Schaff, P. J. Tasker, and L. F. Eastman, IEEE Photonics Tech. Lett. 2, 9 (1990).

${ }^{7}$ G. Arnold, K. Petermann, and E. Schlosser, IEEE J. Quantum Electron. 19, 974 (1983).

${ }^{8}$ G. P. Agrawal and N. K. Dutta, Long Wavelength Semiconductor Lasers (Van Nostrand Reinhold, New York, 1986), pp. 220-238.

${ }^{9}$ D. Marcuse and T. P. Lee, IEEE J. Quantum Electron. 19, 1397 (1983). 